\title{
Numerical Analysis of Surface Tension Gradient Effect on the Behavior of Gas Bubbles at the Solid/Liquid Interface of Steel
}

\author{
Sang-Min LEE, Sang-Joon KIM, ${ }^{*}$ Youn-Bae KANG and Hae-Geon LEE \\ Graduate Institute of Ferrous Technology (GIFT), Pohang University of Science and Technology (POSTECH), San 31, Hyoja- \\ dong, Pohang, 790-784 Korea. E-mail: sjmount@postech.ac.kr, ybkang@postech.ac.kr, hglee@postech.ac.kr
}

(Received on March 15, 2012; accepted on May 14, 2012)

\begin{abstract}
The mechanism which governs the behavior of bubbles in the vicinity of the solidification front where both the concentration and temperature gradients exist was investigated and the effect of the solutal and thermal marangoni forces to the entrapment of bubble were compared and discussed.

When a bubble approaches the solid/liquid interface, it first encounters the thermal boundary layer, which is much thicker than the concentration boundary layer, and experiences the thermal marangoni force. This force, which occurs due to the temperature gradient in the thermal boundary layer, pushes the bubble away from the solidification front if the sulfur concentration is lower than the critical value which was found to be around 47-60 ppm, but pulls it toward the solidification front if the sulfur concentration is higher than the critical value. Only if the bubble passes through the thermal boundary layer successfully, it then arrives at the concentration boundary layer where a strong solutal marangoni force comes into action on the bubble to pull it to the solid/liquid interface.

The above analysis predicts that the bubble-induced surface defect of a cast be strongly affected by the sulfur concentration and there exists a critical sulfur concentration above which the surface defect becomes increasing. The above prediction was validated by the CFD (Computational Fluid Dynamics) simulation and the trial at the commercial casting plant, and they were in good agreement with each other.
\end{abstract}

KEY WORDS: bubble; surface tension gradient; marangoni force; boundary layer; temperature; concentration; sulfur content.

\section{Introduction}

In order to prevent the clogging of the submerged entry nozzle (SEN) in the continuous casting of steel, it has been a usual practice to inject the argon gas into SEN. The argon gas injected forms gas bubbles owing to turbulence of molten steel in the mold. Most of the bubbles float up quickly due to their buoyancy force and are removed from molten steel by passing through the top flux. However, there are some bubbles which stay in the liquid steel for a while and move by following the flow of the liquid steel. During the residence time of a bubble in the molten steel pool, it may attract many fine inclusions to be caught at its surface. Some of this kind of bubble fails to float up to the top, but are attracted to the solidification front in the mold, and finally entrapped at the solidifying shell. These entrapped bubbles become the major cause of defects such as blisters and slivers which appear in the rolled products. ${ }^{1)}$

In order to elucidate the mechanism by which particles or bubbles are captured by the solidification front, a number of studies have been reported previously. ${ }^{2-16)}$ When a particle or bubble touches the solid/liquid interface of steel it may be either entrapped or pushed away. The fate of particles and

\footnotetext{
* Corresponding author: E-mail: sjmount@postech.ac.kr DOI: http://dx.doi.org/10.2355/isijinternational.52.1730
}

bubbles is determined by various conditions such as velocity of solidification front, particle size, conductivity, fluid flow, etc. The critical growth velocity for PET (Pushing Engulfment Transition) was suggested and examined by various methods such as in-situ observation using Confocal Scanning Laser Microscope (CSLM), ${ }^{2,3)}$ microgravity experiment, ${ }^{4)}$ etc. ${ }^{5-7)}$ However, most of the PET studies were carried out with fine solid particles under the stationary state at which the molten steel was mostly still, contrary to the continuous casting process in which the molten steel is of highly turbulent state. The behavior of argon bubbles and inclusions in continuous caster were studied by water model experiments and mathematical models. ${ }^{8,9)}$ Water model was able to show how bubbles move in the casting mold but it is difficult to simulate the solidification, and the mathematical model treated a trap of particles when they touches the solidification front. Yuan and Thomas ${ }^{10)}$ proposed a criterion of the entrapment of solid particles less than $400 \mu \mathrm{m}$ considering ten different forces and turbulent in continuous casting of steel.

Through the basic research on the motion of fine particles under the interfacial tension gradient, Mukai and Lin ${ }^{11}$ derived the force acting on the particle due to the interfacial tension gradient between particle and solution. Wang et $a l .{ }^{12)}$ performed water model experiment to observe the behavior of fine $\mathrm{H}_{2}$ bubbles $(<50 \mu \mathrm{m})$ near the solidifying 
water solution. On the basis of those results, the number of entrapped bubbles are increased due to the high concentration gradient of sulfur, ${ }^{13-16)}$ and it is well-known that sulfur is one of detrimental elements for bubble related defects of the steel product. Thus many steelmakers have tried to reduce sulfur content as low as possible.

Miyake et al. ${ }^{16)}$ investigated the influence of sulfur on the entrapment of large bubble $(\sim 1 \mathrm{~mm})$. However, it still has many ambiguities between prediction and industrial result of bubble entrapment, especially about the temperature effect. Figure 1 shows the dependencies of defect on sulfur content and tundish temperature of ultra-low carbon steel. It is seen in Fig. 1(a) that the defect increases with increase in the sulfur content in the steel, which is well known, but it is also seen that the defect increases more rapidly when the sulfur content exceeds a certain level.

There are hardly any previous studies as to how the temperature of the molten steel and especially the temperature gradient in the vicinity of the solidification front affects the behavior of bubbles. The plant data shown in Fig. 1(b) clearly reveals the effect of temperature on the surface defect.

The purpose of the present study is to clarify the mechanism which governs the behavior of bubbles in the vicinity of the solidification front where both the concentration and temperature gradients exist.

\section{Background}

\subsection{Marangoni Force on the Bubble in the Continuous Casting Mold}

When a liquid surface is subjected to non-uniformity in surface tension, then liquid of high surface tension tends to pull the adjacent liquid of low surface tension, and it is called the marangoni effect. Accordingly liquid flow at the surface sets out. Since the surface tension is a function of solute concentration and temperature, marangoni effect emerges under special circumstances which exhibit a substantial gradient of temperature or solute concentration.

In the continuous casting mold, the concentration gradient due to the rejection of the solutes such as sulfur and oxygen to the liquid from the solid/liquid interface of steel, and the temperature gradient due to the mold cooling of liquid steel are always generated in the vicinity of the solidification front. In the case of bubbles near the solid/liquid interface, therefore, the marangoni effect appears and induces a shear stress on the surface of bubble, which causes marangoni stress.
Mukai and $\operatorname{Lin}^{11)}$ calculated the marangoni force $F_{M a}$ by integrating the marangoni stress over the surface of spherical particle of radius $R$ which is located near the solidifying interface with an surface tension gradient $K$ and derived Eq. (1)

$$
F_{M a}=-\left(\frac{2 \pi}{R}\right) \int_{-R}^{R} K\left(R^{2}-x^{2}\right) d x
$$

where $K$ is the surface tension gradient in the solidification direction $x$. Let's assume that the surface tension gradient $K$ exists only in the narrow region near the interface, so called boundary layer, and is constant over the surface area of particle. When the boundary layer thickness $\delta$ is larger than the bubble diameter $2 R$, that is $\delta>2 R$, so the particle is fully submerged in the boundary layer, then the marangoni force on the particle is calculated simply as Eq. (2)

$$
F_{M a}=-\frac{8}{3} \pi R^{2} K
$$

In the case of small boundary layer thickness, that is $\delta<$ $2 R$,

$$
F_{M a}=-\left(\frac{2 \pi}{R}\right) K \int_{-R}^{-R+\delta}\left(R^{2}-x^{2}\right) d x
$$

The surface tension $\sigma$ is a function of temperature and solute concentration, and then surface tension gradient $K$ can be separated into the temperature dependent term $K_{T}$ and the concentration dependent term $K_{C}$ as the partial derivatives in Eq. (4)

$$
\begin{aligned}
K & =\left(\frac{d \sigma(C, T)}{d x}\right) \\
& =\left[\frac{\partial \sigma(C, T)}{\partial C}\right]_{T}\left(\frac{d C}{d x}\right)+\left[\frac{\partial \sigma(C, T)}{\partial T}\right]_{C}\left(\frac{d T}{d x}\right)=K_{C}+K_{T}
\end{aligned}
$$

The surface tension gradient $K$ is the sum of thermal and solutal terms, $K_{T}$ and $K_{C}$. In the previously reported works, however, $K$ has been assumed to be the same as $K_{C}$ because $K_{T}$ is generally much smaller than $K_{C}$ in liquid iron. ${ }^{9,12-16)}$

With the basic consideration described above, which neglects the effect of temperature, more precisely the effect of temperature gradient, it is difficult to interpret the observations shown in Fig. 1, which shows the sudden change in the rate of defects at a certain sulfur concentration and the strong influence of temperature on the defect. There are two different boundary layers developed in the liquid side in front of the liquid/solid interface: namely, the concentration
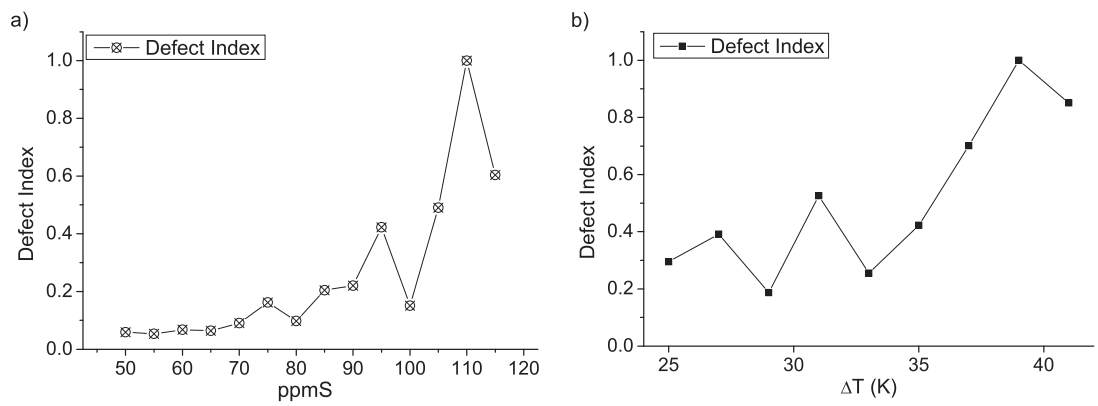

Fig. 1. Number distribution of bubble-related defects of ultra-low carbon steel depends on a) sulfur concentration and b) superheat. 
boundary layer and thermal boundary layer. The important parameters which govern the behavior of a bubble within these layers will be the respective thickness of these boundary layers and the size of the bubble for a given temperature and sulfur concentration of the system (bulk liquid steel). It is, therefore, important to carefully determine the forces exerting on the bubble of a given size in each of these boundary layers. For example, if the thermal boundary layer is much thicker than the concentration boundary layer, then a bubble coming from the liquid bulk will first encounter the thermal boundary layer before meeting the concentration boundary layer. Therefore, whether or not the bubble will eventually enter the concentration boundary layer will be determined by the force balance in the thermal boundary layer. If this is the case, $K_{T}$, albeit small, will play the pivotal role on the movement of the bubble in the thermal boundary layer.

\subsection{Surface Tension Model}

In many cases, the surface tension model of liquid Fe- $i$ binary system which is containing surface active element has been given by a combination of Gibbs and Langmuir adsorption isotherms model proposed by Belton ${ }^{17)}$ as shown in Eq. (5).

$$
\sigma^{P}-\sigma=R T \Gamma_{i} \ln \left(1+K_{a d} a_{i}\right)
$$

where, $\sigma^{P}$ is the surface tension of pure liquid metal, $R$ gas constant, $\Gamma_{i}$ surface adsorption of solute $i$ at saturation, $K_{a d}$ adsorption coefficient, and $a_{i}$ activity of solute $i$.

Sahoo et al. ${ }^{18)}$ later modified the above equation to include the effect of temperature on the adsorption coefficient $\left(K_{a d}\right)$ as follows:

$$
\begin{aligned}
& K_{a d}=k_{i} e^{-\left(\Delta H^{0} / R T\right)} \\
& \sigma=\sigma_{0}-A\left(T-T_{m}\right)-R T \Gamma_{i} \ln \left(1+k_{i} a_{i} e^{-\left(\Delta H^{0} / R T\right)}\right) \ldots
\end{aligned}
$$

where, $A$ is the temperature coefficient of surface tension of pure iron, $T_{m}$ the melting temperature of iron, $k_{i}$ is a constant related to the entropy of sulfur segregation, and $\Delta H^{0}$ the standard heat of adsorption. The parameters of $\mathrm{Fe}-\mathrm{S}$ system which were used in present study are listed in Table 1. The

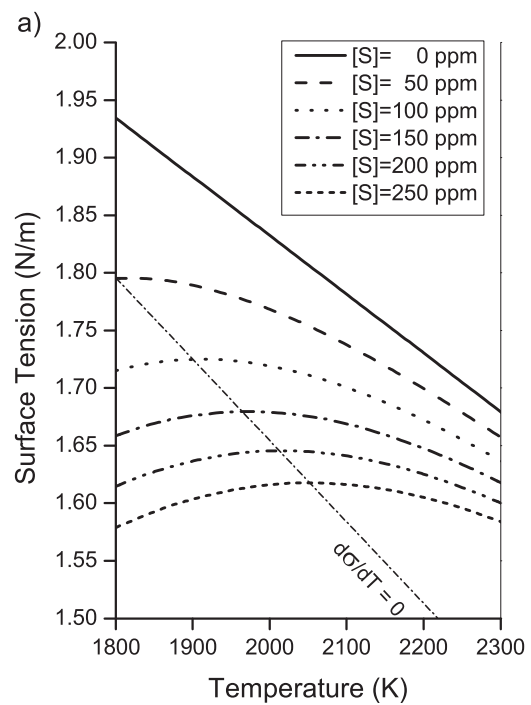

surface tension of $\mathrm{Fe}-\mathrm{S}$ binary system with respect to temperature and sulfur concentration is plotted by Eq. in Fig. 2(a). The temperature coefficient of surface tension $(d \sigma / d T)$ in Eq. (4) can be obtained by differentiating Eq. (7) with respect to temperature.

$$
\frac{\partial \sigma}{\partial T}=-A-R \Gamma_{i} \ln \left(1+K_{a d} a_{i}\right)-\frac{K_{a d} a_{i}}{\left(1+K_{a d} a_{i}\right)} \frac{\Gamma_{i} \Delta H^{0}}{T}
$$

As shown in Fig. 2(b), a slope of surface tension, $d \sigma / d T$, varies as the sulfur concentration changes. It is seen that the surface tension of pure iron decreases with increase in temperature. As the sulfur concentration becomes higher, however, the surface tension increases with temperature and then decreases. This tendency was more intensively reported by Keene. ${ }^{19)}$

Near the melting temperature of iron $(1809 \mathrm{~K})$, the critical sulfur concentration at which the sign of $d \sigma / d T$ changs is about $47 \mathrm{ppm}$. The critical sulfur concentration increases with increasing temperature as shown in Fig. 2(b), and the critical sulfur concentration varies from 47-60 ppm range for the temperature of $1809-1840 \mathrm{~K}$.

\subsection{Boundary Layer Thickness}

The ratio of boundary layer thicknesses of convective heat and mass transport can be expressed briefly by using Lewis number, Le, which consists of Prandtl number, $\operatorname{Pr}$ and Schmmit number, Sc.

$$
L e=S c / P r=\alpha / D_{s}
$$

where $\alpha$ is thermal diffusivity, $D_{s}$ is mass diffusivity of solute. In the case of liquid Fe-S binary system, $\alpha$ and $D_{s}$ are $6.1 \times 10^{-6} \mathrm{~m}^{2} / \mathrm{s}^{20)}$ and $3.4 \times 10^{-9} \mathrm{~m}^{2} / \mathrm{s},{ }^{13)}$ respectively. The value of $L e$ of liquid $\mathrm{Fe}-\mathrm{S}$ binary system is then about $10^{3}$. It implies that the thickness of the thermal boundary layer is

Table 1. Parameter values used in calculating surface tension of Fe-S binary system. ${ }^{18)}$

\begin{tabular}{ccccccc}
\hline $\begin{array}{c}\sigma_{0} \\
(\mathrm{~N} / \mathrm{m})\end{array}$ & $\begin{array}{c}A \\
(\mathrm{~N} / \mathrm{mK})\end{array}$ & $\begin{array}{c}R \\
(\mathrm{~J} / \mathrm{mol} \mathrm{K})\end{array}$ & $\begin{array}{c}T_{m} \\
(\mathrm{~K})\end{array}$ & $\begin{array}{c}\Gamma \mathrm{s} \\
\left(\mathrm{mol} / \mathrm{m}^{2}\right)\end{array}$ & $k_{\mathrm{s}}$ & $\begin{array}{c}\Delta H^{0} \\
(\mathrm{~J} / \mathrm{mol})\end{array}$ \\
\hline 1.94 & 0.00051 & 8.314 & 1810 & $1.30 \times 10^{-5}$ & 0.00318 & $-1.66 \times 10^{5}$
\end{tabular}

b)

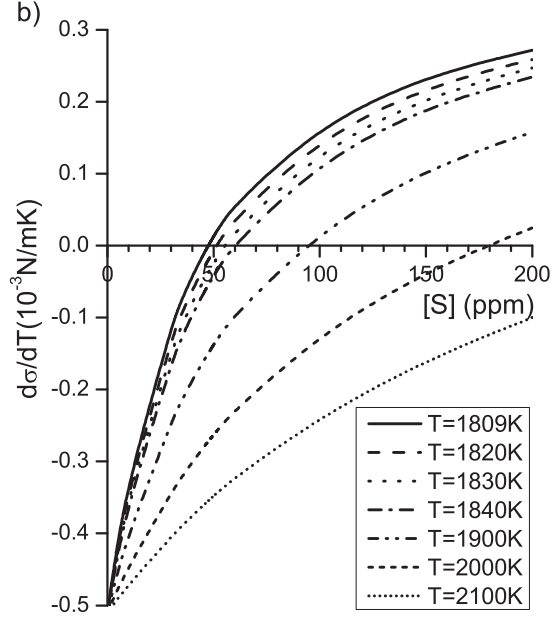

Fig. 2. a) surface tension and b) $d \sigma / d \mathrm{~T}$ of $\mathrm{Fe}-\mathrm{S}$ system vary with sulfur concentration and temperature. 
much larger than that of the solutal boundary layer.

When the solidification without convective flow is assumed, the liquid concentration in diffusion process at steady-state can be calculated by using following equation, ${ }^{21)}$

$$
C_{L}=C_{0}\left(1+\frac{1-k}{k} e^{-\left(R_{g} / D_{L}\right) x}\right)
$$

where, $C_{L}$ and $C_{0}$ are the liquid concentration of the position $x$ from the interface and the bulk, respectively, $k$ is the equilibrium partition coefficient, $R_{g}$ is the interface growth rate, $D_{L}$ is the mass diffusivity in the liquid melts. $D_{L}$ and $k$ for the liquid $\mathrm{Fe}-\mathrm{S}$ binary system are $3.4 \times 10^{-9} \mathrm{~m}^{2} / \mathrm{s}$ and $0.05{ }^{13}$ ) and in the continuous casting mold, the growth rate of solidification front is about $1.3 \times 10^{-3} \mathrm{~m} / \mathrm{s}^{22}$ )

The boundary layer thickness is defined as the distance between the surface and the position $x$ where $C_{L}$ becomes $99 \%$ of the bulk concentration $C_{0}$, it can be calculated by using Eq. (10) as about $12 \mu \mathrm{m}$. If the forced convection is taken into account, the solutal boundary layer thickness should be smaller. On the other hand, the thermal boundary layer is developed much broader than the solutal boundary layer as the $L e$ in the liquid Fe-S binary system is order of $10^{3}$.

\section{Thermal and Solutal Marangoni Force Calculation}

The entrapment of a bubble follows 3 steps as shown schematically in Fig. 3. Step 1 is the bubble approaching the thermal boundary layer. In this step, the bubble should move toward the solidifying interface from the bulk liquid. During this step, movement of the bubble is mainly controlled by the drag and buoyancy forces in the bulk liquid flow.

Once the bubble has entered the boundary layer, it is subjected to, in addition to the drag and buoyancy forces, the

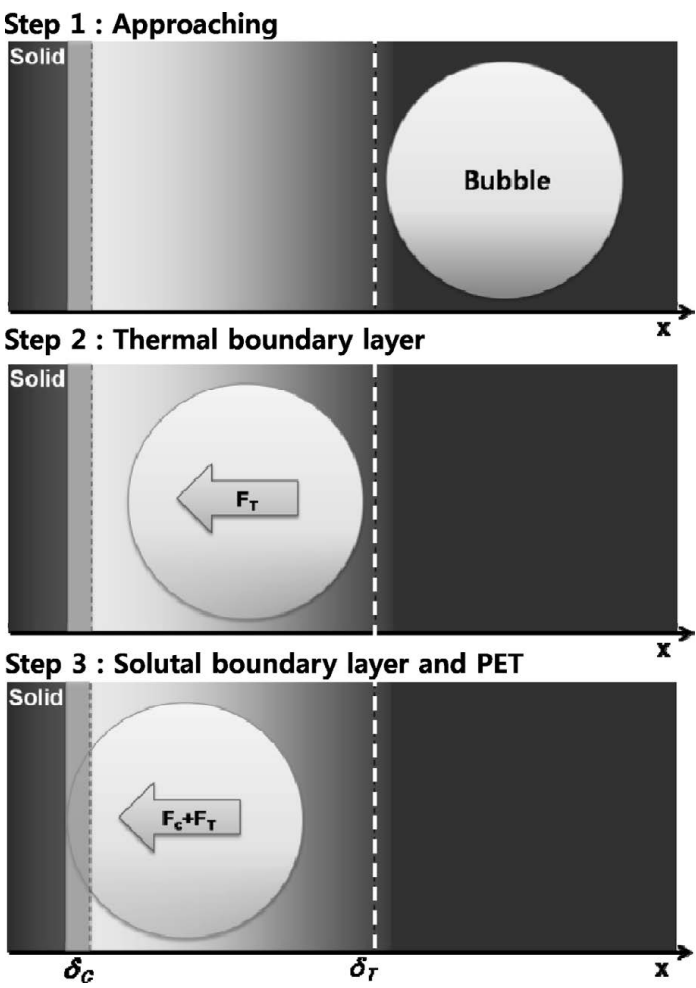

Fig. 3. Schematic steps of bubble entrapment. surface tension gradient force (marangoni force) due to the gradient of temperature and also due to the gradient of concentration of the solute in the boundary layer (Step 2). As discussed previously, the thickness of the thermal boundary layer is much larger than that of concentration, and thus the thermal boundary layer is the first interaction zone which the bubble encounters. Hence, in Step 2, the behavior of the bubble is affected mainly by the thermal marangoni force.

Referring to Fig. 2, which is the result of Eqs. (7) and (8), the direction of the thermal Marrangoni force, i.e., the sign of $d \sigma / d T$, is determined by both prevailing temperature and sulfur concentration. Therefore, the bubble in Step 2 may be either pushed away from the solidification front (i.e., when $d \sigma / d T<0$ ), or pulled toward the solidification front (i.e., when $d \sigma / d T>0$ ). In Fig. 2(b), it is seen that $d \sigma / d T$ is always negative for the sulfur concentration lower than the critical concentration of $d \sigma / d T=0$, which means that bubbles always receive the marangoni force which pushes the bubbles away from the solidification front. If this is the case, bubbles can hardly be attached to the solid shell, reducing bubbleinduced defects. If the sulfur concentration is higher than the critical sulfur concentration, on the other hand, there is a critical temperature for given sulfur concentration, below which $d \sigma / d T$ is positive, meaning that bubbles are attracted toward the solidification front. The line which connects the points of $d \sigma / d T=0$ is shown in Fig. 2(a).

Once the net force on the bubble in Step 2 pulls it toward the interface, it meets the solutal boundary layer in Step 3. In this step, there exist both solutal and thermal boundary layers, and so they are applied to the bubble simultaneously. Since the solutal marangoni force is always in the direction toward the interface, the bubble, once enters the solutal boundary layer, is attached to the interface in Step 3.

The bubble which has arrived at the interface after passing through Step 2 and 3 may either be engulfed into the solidifying shell or stay at the interface, moving along with the solidification front. The Pushing Engulfment Transition (PET) model $^{2-7)}$ suggests that the bubble/particle at the interface is engulfed into the solid shell when the growth rate of the shell exceeds the critical rate. The initial growth rate of the shell in the steel continuous casting is much higher than the critical rate, ${ }^{22)}$ most bubbles which touch the solidifying interface at the initial solidifying shell are engulfed and become the cause of surface defects.

Among the 3 steps abovementioned, Step 2 plays the pivotal role which determines whether the bubble will be entrapped in the solid shell or pushed away from the solidifying interface.

In order to evaluate the magnitude of solutal and thermal marangoni forces, the effective area $A$ of the bubble is defined by using Eqs. (2) and (3). $K$

$$
F_{M a}=A K
$$

In the case of $\delta<2 R$,

$$
A=-\left(\frac{2 \pi}{R}\right) \int_{-R}^{-R+\delta}\left(R^{2}-x^{2}\right) d x
$$

And in the case of $\delta>2 R$,

$$
A=-\frac{8}{3} \pi R^{2}
$$


The effective area of the bubble is calculated using Eqs. (12) or (13) depending on the thickness of the boundary layer and the diameter of bubbles. The diameter of bubbles which cause defect is much larger than the thickness of the solutal boundary layer $(>1 \mathrm{~mm})$, so that the effective area by the solutal boundary layer, $A_{C}$ is calculated by using Eq. (12). $A_{T}$ is calculated from Eq. (12) when the diameter of bubble is larger than the thermal boundary layer thickness, otherwise, calculated by using Eq. (13).

$\mathrm{Toh}^{23)}$ calculated the maximum thickness of the velocity boundary layer up to $3 \mathrm{~mm}$ in the continuous casting mold. For the materials which has low Prandtl number $(<1)$ such as liquid metal, the ratio of the thickness of temperature and velocity boundary layer, $\Delta$ is given by Schlichting ${ }^{24)}$ as follows.

$$
\frac{3}{10} \Delta^{2}-\frac{3}{10} \Delta+\frac{2}{15}-\frac{3}{140} \frac{1}{\Delta^{2}}+\frac{1}{180 \Delta^{3}}=\frac{37}{315} \frac{1}{\operatorname{Pr}} .
$$

Prandtl number of liquid iron is about 0.14 , and then $\Delta$ is about 2 . Hence the thickness of the thermal boundary layer becomes $6 \mathrm{~mm}$ at most, and it can be reasonably assumed that most of detrimental size bubbles are to be fully enclosed in the thermal boundary layer.

The thickness of the solutal boundary layer $\delta_{C}$ is changed by the solid growth rate and fluid flow. Applying faster growth rate at the initial solidification to Eq. (10), $\delta_{C}$ is calculated to be small. And Miyake ${ }^{16)}$ calculated the thickness of solutal boundary layer to be $21.5 \mu \mathrm{m}$ in the case of $0.1 \mathrm{~m} / \mathrm{s}$ of molten steel velocity. Considering the faster growth rate and the result of Miyake, ${ }^{16)} 10 \mu \mathrm{m}$ and $20 \mu \mathrm{m}$ were used as the value of $\delta_{C}$.

Figure 4 shows the ratio of the thermal to solutal effective area $\left(A_{T} / A_{C}\right)$ with varying bubble diameter $D$. When $\delta_{C}=10 \mu \mathrm{m}$ and $D=1000 \mu \mathrm{m}$, the thermal effective area $A_{T}$ is about 3000-4000 times larger than the solutal area and it reaches more than $10^{4}$ times when bubble diameter is $2 \mathrm{~mm}$. It means that the larger bubbles are affected more by the thermal marangoni. Therefore the thermal marangoni force, which has been neglected in the previous works mainly because of the small surface tension gradient, has considerable significance when the thermal effective area $A_{T}$ acting on the large bubble is taken into account.

The surface tension gradient $K$ can be calculated by using Eqs. (4) and (7) under boundary conditions shown in Fig. 5. The solutal boundary layer is much thinner and confined

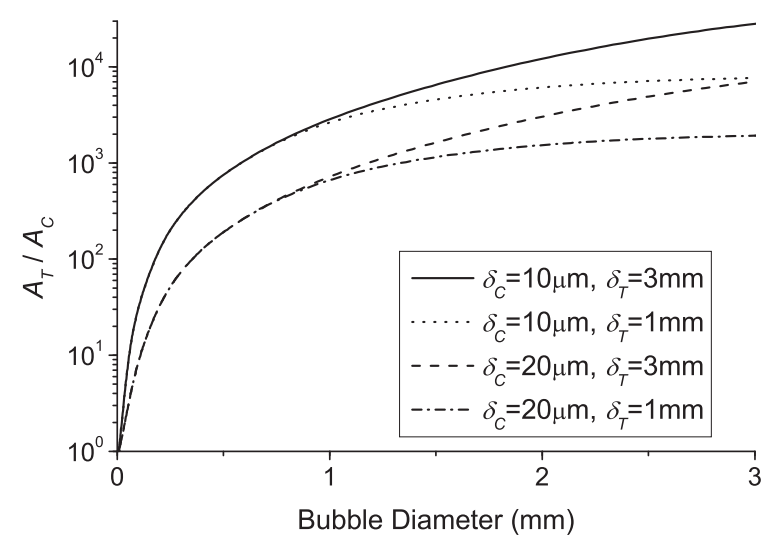

Fig. 4. The ratio of the thermal to the solutal effective area, $A_{T} / A_{c}$. closer to the interface. The concentration gradient of sulfur exists only in the very narrow region near the solidification front, and thus the temperature in this region can be assumed constant at the liquidus temperature $T_{L}$. The sulfur concentration in the thermal boundary layer is assumed to be the same as the bulk sulfur concentration.

Assuming that profiles of concentration and temperature are linear as shown in Fig. 5, the surface tension gradients of the concentration and the temperature, $K_{C}$ and $K_{T}$ are respectively,

$$
K_{C}=\left(\frac{d \sigma(C, T)}{d x}\right)_{T}=\left[\frac{\Delta \sigma(C)}{\delta_{c}}\right]_{T=T_{L}}=\left(\frac{\sigma\left(C_{0}, T_{L}\right)-\sigma\left(C_{L}^{*}, T_{L}\right)}{\delta_{c}}\right)
$$

$$
K_{T}=\left(\frac{d \sigma(C, T)}{d x}\right)_{C}=\left[\frac{\Delta \sigma(T)}{\delta_{T}}\right]_{C=C_{0}}=\left(\frac{\sigma\left(T_{0}, C_{0}\right)-\sigma\left(C_{0}, T_{L}\right)}{\delta_{T}}\right)
$$

$$
C_{L}^{*}=\frac{k_{e f f}}{k} \cdot C_{0}
$$

$$
k_{\text {eff }}=\frac{k}{k+(1-k) \exp \left(-R_{g} \cdot \delta_{C} / D_{L}\right)}
$$

where $C_{L}^{*}$ is the concentration of the liquid at the solidifying interface, and $k_{\text {eff }}$ is the effective partition coefficient considering the effect of convection. ${ }^{25}$ )

Figure 6 shows the ratio of the thermal to the solutal surface tension gradient $\left(K_{T} / K_{C}\right)$ as a function of sulfur concentration by Eqs. (15) and (16). The negative value in the figure means that the directions of the thermal and solutal marangoni forces are opposite to each other. The result changes with sulfur concentration but it is very small as $10^{-4}$ order. As discussed, the effect of temperature gradient has been ignored since $K_{T}$ is much smaller than $K_{C}$, but, the marangoni force is the product of the effective area $A$ and the surface tension gradient $K$ as shown in Eq. (11). Hence, the high $A_{T}$ of $1 \mathrm{~mm}$ order diameter compensates the low $K_{T}$ and it results in the comparable strength of the thermal marangoni force.

Figures 7 and $\mathbf{8}$ show calculated results of the solutal and thermal marangoni force as a function of the sulfur content of the steel. The negative values imply that the direction of

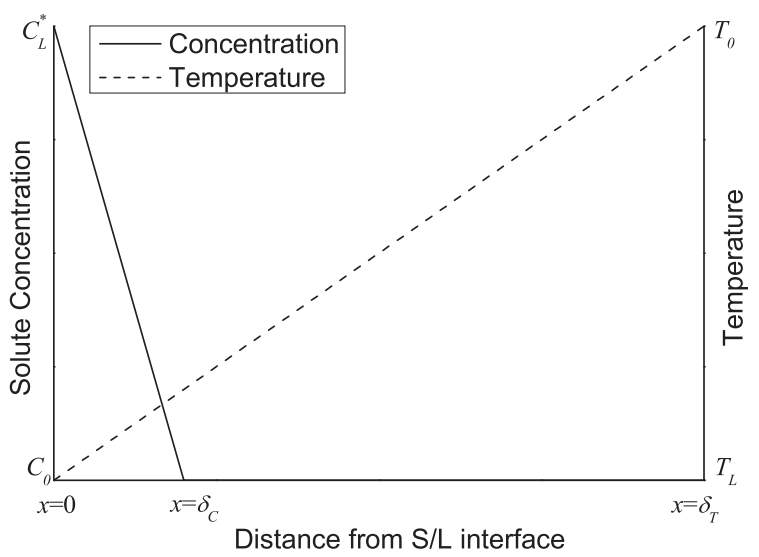

Fig. 5. Schematic profile of the concentration and temperature. 


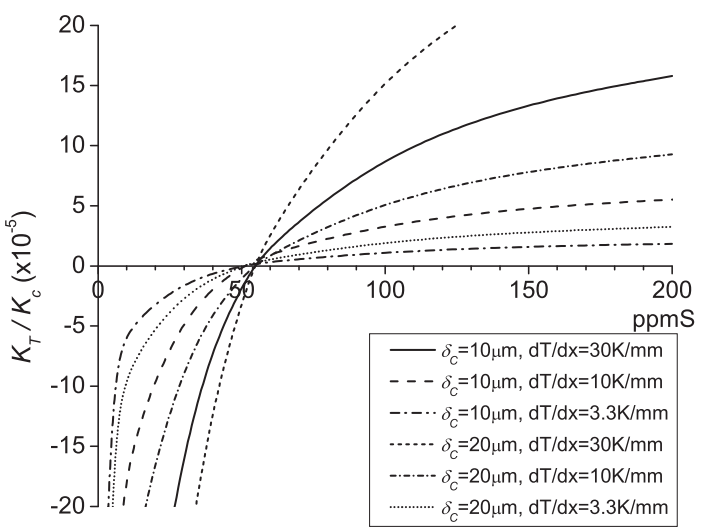

Fig. 6. Ratio of the thermal to the solutal surface tension gradient $\left(K_{T} / K_{c}\right)$ as a function of sulfur concentration.

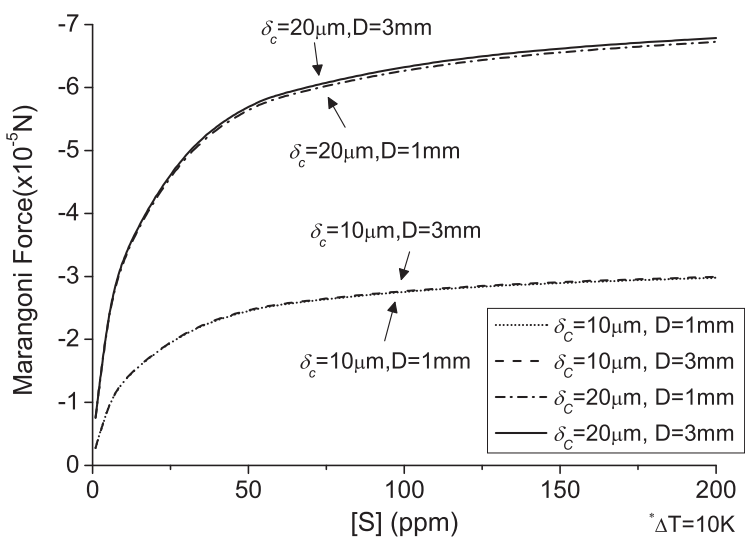

Fig. 7. The calculated solutal Marangoni force, $F_{C}$.

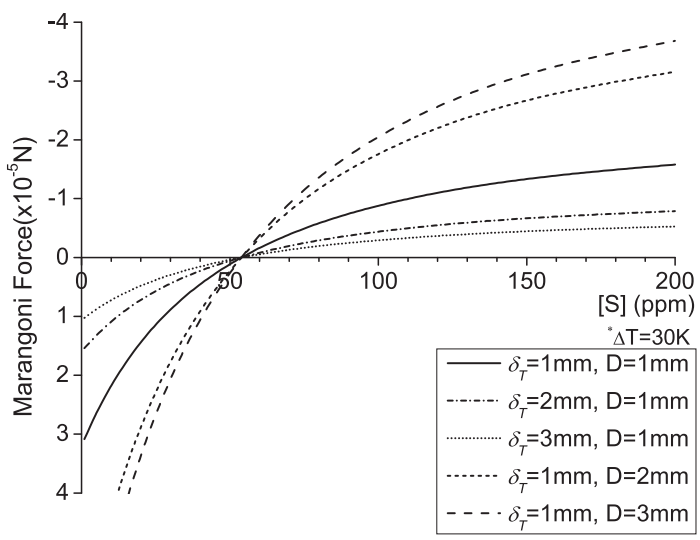

Fig. 8. The calculated thermal marangoni force, $F_{T}$.

the force is to the solidifying interface.

In Fig. 7, the solutal marangoni force $\left(F_{C}\right)$ increases with the sulfur content. In particular, $F_{C}$ increases drastically up until the sulfur content reaches about $50 \mathrm{ppm}$, and then the increase slows down for further increase in sulfur. It can also be noted that the bubble diameter have little effect, but the thickness of the solutal boundary layer, $\delta_{C}$, exerts a significant effect. When $\delta_{C}$ is doubled from $10 \mu \mathrm{m}$ to $20 \mu \mathrm{m}, F_{C}$ is also doubled in figure. This is because that, when $\delta_{C}$ is doubled from $10 \mu \mathrm{m}$ to $20 \mu \mathrm{m}$, the solutal surface tension gradient $K_{C}$ becomes the half (see Eq. (15)), but $A_{C}$ becomes 4 times greater (see Eq. (12)). Therefore, $F_{C}$, which is the product of $K_{C}$ and $A_{C}$ as seen in Eq. (11), is doubled finally.
Therefore $F_{C}$ in Fig. 7 shows the two groups according to $\delta_{C}$ of $10 \mu \mathrm{m}$ to $20 \mu \mathrm{m}$.

On the other hand, $F_{T}$, as seen in Fig. 8 , changes from the positive to negative value as the sulfur content increases. This implies that, when the sulfur content is low, the thermal Marnagoni force pushes the bubble away from the solidification front, whereas it pulls the bubble toward the solidification front as the sulfur content is excessive. The change in the sign of $F_{T}$ occurs at $54 \mathrm{ppm}$ of the critical sulfur concentration, which is because the sign of $d \sigma / d T$ is changed as shown in Fig. 2(b). Hence, $F_{T}$ plays a role of pushing (positive) at low sulfur and pulling (negative) at high sulfur. Unlike $F_{C}$, it can also be noted that the larger bubble diameter and the smaller $\delta_{T}$, the stronger $F_{T}$ is. It is because the larger $D$ and the smaller $\delta_{T}$ increase both $A_{T}$ and $K_{T}$.

In order to compare the solutal and thermal marangoni forces $\left(F_{C}\right.$ and $\left.F_{T}\right)$ shown in Figs. 7 and 8, sample calculations were made for the cases that $\delta_{C}, \delta_{T}$ and superheat $(\Delta T)$ are $10 \mu \mathrm{m}, 1 \mathrm{~mm}$ and $30 \mathrm{~K}$ respectively, and the results are given in Fig. 9. In the figure, $F_{C}$ is only for $D=1 \mathrm{~mm}$ case since influence of the bubble diameter on $F_{C}$ is negligible. It is reminded that $F_{C}$ is negative for all range of sulfur concentrations, meaning that $F_{C}$ always attracts the bubble toward the interface. In the case of $F_{T}$, it changes from positive to negative at $54 \mathrm{ppm}$ of sulfur content, which means that the thermal marangoni force actually pushes the bubble away from the interface at less than $54 \mathrm{ppm}$ of sulfur content while it pulls the bubble toward the interface with sulfur over $54 \mathrm{ppm}$. Notice that $F_{T}$ increases with the bubble diameter, and thus $F_{T}$ affects more influence on a larger bubble.

In contrast, $F_{C}$ is virtually bubble-size independent. In Fig. 9, the surface defect index given in Fig. 1(a) is superimposed, which shows a drastic increase in defect when sulfur content reaches about $70 \mathrm{ppm}$. The sudden increase of defect at and above the sulfur concentration can be interpreted as follows:

(1) When a bubble enters the thermal boundary layer which is much wider than the concentration boundary layer, it should experience either a force pulling toward the solidification front or a force pushing away from it, depending on the sulfur concentration.

(2) When the sulfur concentration is lower than about $54 \mathrm{ppm}, F_{T}$ is positive, meaning that the bubble is pushed away when it enters the thermal boundary layer. This bubble will move away without even experiencing the solutal marangoni force $\left(F_{C}\right)$ as the concentration boundary layer is much thinner than the thermal boundary layer. This is the reason why the number of defects found in the plant trial is very low when the sulfur concentration is lower than about $54 \mathrm{ppm}$.

(3) When the sulfur concentration is higher than about $54 \mathrm{ppm}$, on the other hand, $F_{T}$ becomes negative, meaning that the bubble entering the thermal boundary layer is pulled toward the solidification front. The bubble will continue to move toward the front and eventually enter the concentration boundary layer in which the solutal marangoni force $\left(F_{C}\right)$ begins to exert the pulling force which is much stronger than $F_{T}$ in the practical sulfur concentration range as seen in Fig. 9. 


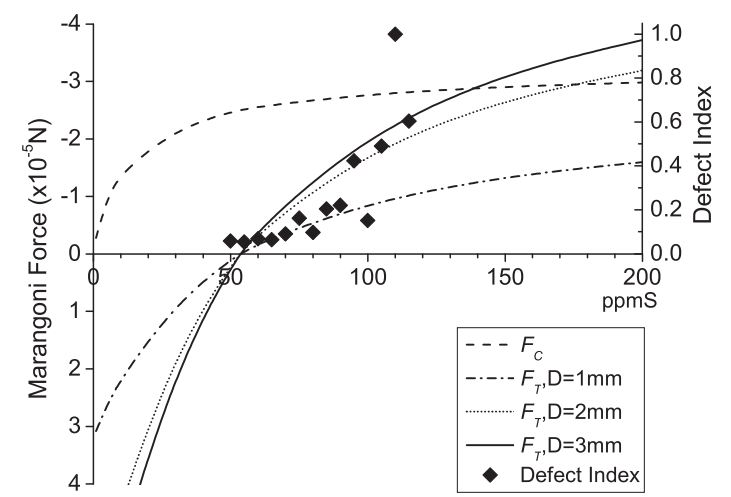

Fig. 9. The results of the marangoni force calculation in case of $\delta_{c}=10 \mu \mathrm{m}, \delta_{T}=1 \mathrm{~mm}$, and $\Delta \mathrm{T}=30 \mathrm{~K}$.

Besides the sulfur content of steel, the defect index tends to increase with temperature as seen earlier in Fig. 1(b). Figure 10 shows how the superheat of steel affects the marangoni forces $\left(F_{C}\right.$ and $\left.F_{T}\right)$ at different sulfur concentrations. The calculations are based on $\delta_{C}=10 \mu \mathrm{m}, \delta_{T}=1 \mathrm{~mm}$, and $D=2 \mathrm{~mm}$. As expected, $F_{C}$ is little influenced by superheat. However, $F_{T}$ is significantly influenced by both the superheat and sulfur concentration. If the sulfur concentration is lower than $54 \mathrm{ppm}, F_{T}$ becomes more positive as the superheat increases. This implies that, the higher the superheat, the stronger the $F_{T}$ pushing bubbles away from the surface. On the other hand, if the sulfur concentration is higher than $54 \mathrm{ppm}, F_{T}$ becomes to act in the opposite way: the higher the superheat, the stronger the $F_{T}$ pulling bubbles toward the surface.

\section{CFD Calculation}

\subsection{Marangoni Flow Calculation around a Bubble}

When a bubble (or a particle) is exposed to the field in which the surface tension gradient exists, the force for marangoni flow develops around the bubble (particle) surface. The marangoni flow developed on the surface of a bubble was computed using the commercial CFD package FLUENT ${ }^{\circledR}$. In the simulation, the bubble surface in liquid iron is assumed a rigid slip wall in the $2 \mathrm{D}$ axisymmetric spherical domain. Results are given in Fig. 11, in which Figs. 11(a) and 11(b) are for the sulfur concentration of $30 \mathrm{ppm}$ and $100 \mathrm{ppm}$, respectively. In the figures, shown are the variations of the tangential velocity of liquid iron which is marangoni flow. In the computation, the temperature gradient of $1 \mathrm{~K} / \mathrm{mm}$ was applied.

In the figure it is clearly seen that, when the sulfur concentration is low ( $30 \mathrm{ppm} \mathrm{S}$ ), the tangential velocities of liquid iron around the bubble are all directed toward the solidification front ( $1810 \mathrm{~K}$ left wall) due to the higher surface tension of lower temperature side, meaning that the bubble is to be pushed away from the front (Fig. 11(a)) as derived in Eq. (2) by Mukai and Lin. ${ }^{11)}$ On the other hand, if the sulfur concentration is high (100 ppm S), the opposite is the case; i.e., the velocity vectors are directed away from the solidification front, meaning that the bubble is attracted toward the front (Fig. 11(b)).

The total viscous force exerting on the bubble can be obtained by integrating the marangoni stress found through

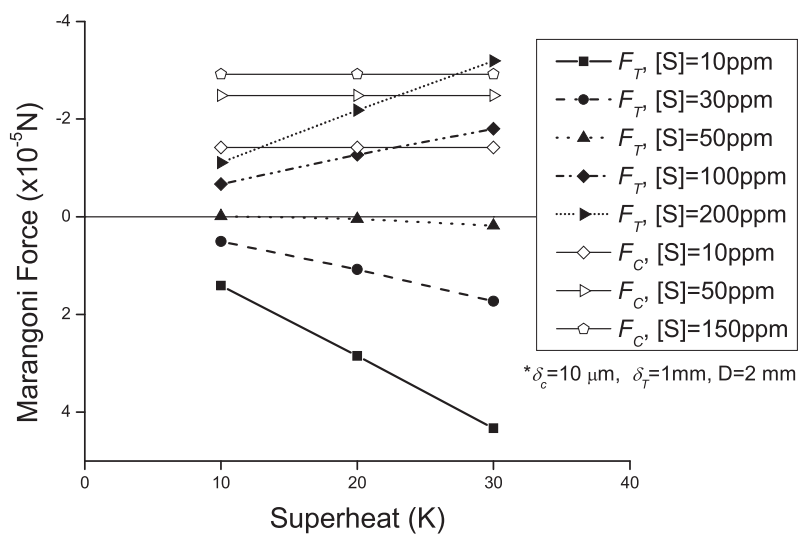

Fig. 10. Relationship between the superheat of steel and the marangoni forces in the case of $\delta_{c}=10 \mu \mathrm{m}, \delta_{T}=1 \mathrm{~mm}$, and $D=2 \mathrm{~mm}$.

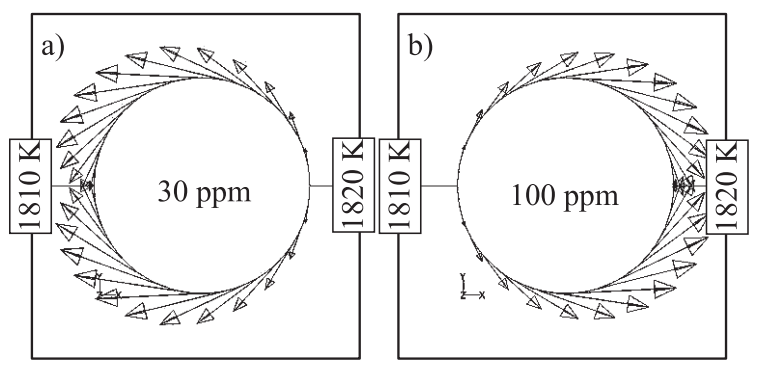

c)

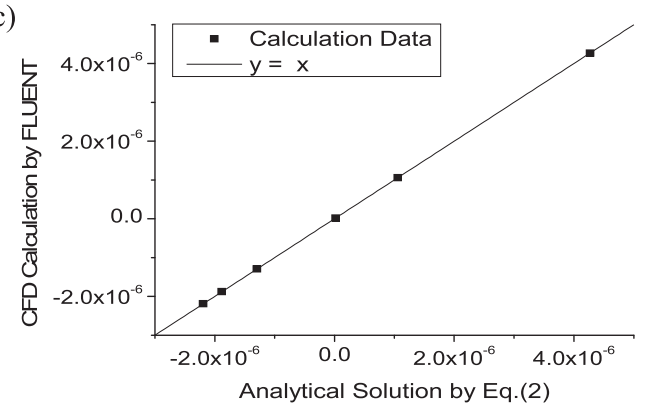

Fig. 11. The marangoni flow calculation around a bubble: the velocity vector field of the marangoni flows on the bubble surface in the liquid iron at a) $30 \mathrm{ppm}$ and b) $100 \mathrm{ppm}$ of sulfur, and c) comparison of results by FLUENT simulation and Eq. (2).

the CFD simulation on the bubble surface. The other way, which is simpler, is to calculate using Eq. (2). The results obtained by two different ways are compared in Fig. 11(c). It shows that these two are in good agreement with each other, and thus subsequent calculations are carried out by treating the marangoni force acting on the bubble as the integrated body force by Eqs. (11) and (13).

\subsection{D CFD Simulation of the Bubble Motion}

In order to validate the thermal marangoni model for the gas bubble entrapment during the steel solidification, the numerical 2-dimensional simulation is carried out by using commercial CFD package FLUENT. Fluid flow, distribution of sulfur and temperature, and behavior of bubbles in the 2D rectangular domain are computed. Figure $\mathbf{1 2}$ shows the scheme of computational domain. The domain of computation includes $2 \mathrm{~mm}$ of the flow entry region and $18 \mathrm{~mm}$ of solidifying region along with vertical direction. 2-dimensional 


\section{Flow inlet}

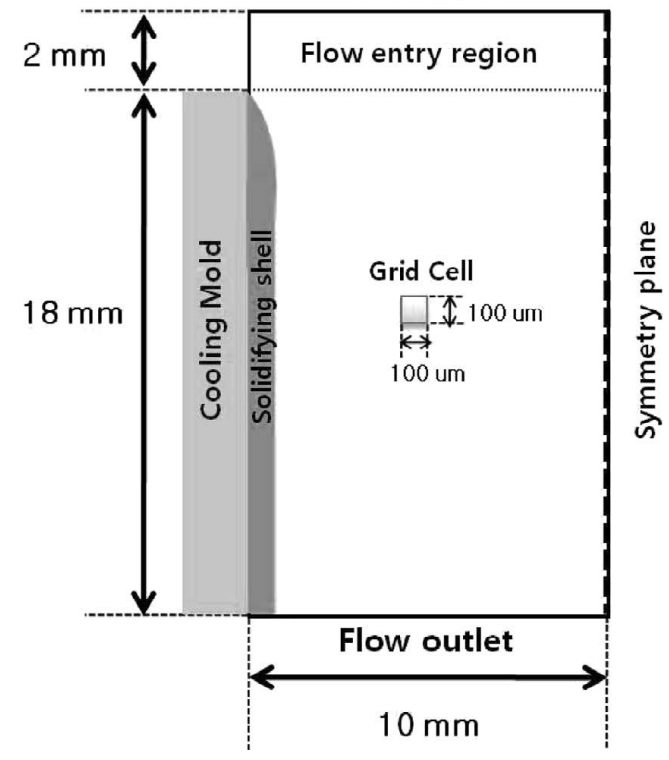

Fig. 12. Scheme of the calculation domain.

laminar fluid velocities, temperature and sulfur species distribution were obtained by solving the governing equations of momentum, energy and species transport under appropriate initial and boundary conditions. The solidification/melting model of Fe-S binary system was also solved by enthalpyporosity technique to acquire appropriate sulfur and temperature distribution at solid/liquid interface.

Temperature of $10 \mathrm{~K}$ over the liquidus temperature of the steel and bulk sulfur concentration was used for initial condition of the simulation. The temperature at the inlet and outlet were assumed the same as the tundish temperature and the mean temperature respectively. The sulfur concentration both at the inlet and at the outlet was fixed to be the same as the bulk sulfur concentration. The convective heat transfer for the solidification was set to the cooling mold wall on the left side of the domain. The amount of sulfur rejected to the liquid during solidification was calculated by the equilibrium partition coefficient of the Fe-S binary system. Fluid properties and computational conditions are listed in Table 2.

After getting reached steady-state of the solidification, Bubble transport is solved by integrating following force balance on the bubble in a Lagrangian reference frame.

$$
\frac{d \mathbf{u}_{B}}{d t}=F_{D}\left(\mathbf{u}-\mathbf{u}_{B}\right)+\frac{\mathbf{g}\left(\rho_{B}-\rho\right)}{\rho_{B}}+\frac{\mathbf{F}_{M a}}{m_{B}}
$$

where,

$$
\begin{gathered}
F_{D}=\frac{18 \mu}{\rho_{B} d_{B}^{2}}\left(1+0.186 \mathrm{Re}^{0.653}\right) \\
\mathbf{g}=\left(0,-9.8 \mathrm{~m} / \mathrm{s}^{2}\right) \ldots \ldots \ldots \ldots \ldots . . . \\
\operatorname{Re}=\frac{d_{B}\left|\mathbf{u}-\mathbf{u}_{p}\right|}{v} \ldots \ldots \ldots \ldots \ldots
\end{gathered}
$$

Those three terms in right-hand side of Eq. (19) are the drag force, buoyancy force and marangoni force respectively. The marangoni force in Eq. (19) is calculated by Eqs. (4), (7), (8), (11) and (13). The gradient of concentration and temperature is obtained from the result of the CFD simula-
Table 2. Properties and conditions of computation.

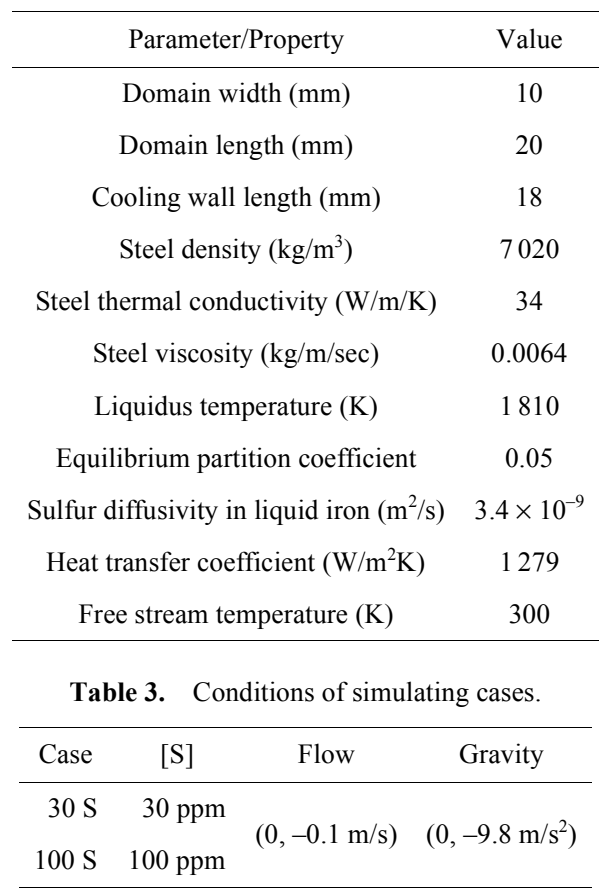

tion. A number of gas bubbles were introduced into the computational domain. Bubbles were treated as a discrete spherical body. The boundary conditions for the discrete second phase were set as escape on the top inlet and bottom outlet.

2 cases of the simulation take place as their different simulating conditions. Table 3 shows conditions of 2 cases which are separated as bulk sulfur concentration. Figure 13 shows results of simulations. The line in the computing domain means the contour line of temperature.

Both Case $30 \mathrm{~S}$ and Case $100 \mathrm{~S}$ in Fig. 13 have the inlet fluid flow of $0.1 \mathrm{~m} / \mathrm{s}$ at the top of the computing domain with the gravity, so that the buoyancy force and the drag force from the fluid flow are acting on the bubble. Additionally the steel was solidifying from the left side wall, so that gradients of the sulfur concentration and the temperature are generated by the sulfur rejection from the solid to the liquid steel and the cooling from the left wall. Thus both the solutal and the thermal marangoni force were considered on the bubble transport.

As shown in Fig. 13, the entrapment behavior of the bubble is mostly determined by the boundary layer, especially the thermal one. The profiles of the boundary layers are plotted in Fig. 14, and it shows the thermal boundary layer thickness is about $2.5 \mathrm{~mm}$ and the solutal one is about $0.07 \mathrm{~mm}$. As discussed in Eq. (9), the Lewis number shows the thermal boundary layer is much larger than the concentration one briefly, hence, the ratio of the thermal to the solutal boundary layer thickness in Fig. 14 is quite reliable.

Groups of bubbles were injected into the computing domain from 3 different positions which stand for each steps of bubble entrapment in Fig. 3 respectively. Each group has 10 bubbles whose diameters from 0.1 to $2.1 \mathrm{~mm}$ are evenly distributed. The first bubble group injected inside the sulfur concentration boundary layer which stands for Step 3 held their initial position and stick on the sold/liquid interface. The bubbles of the second group only in the thermal boundary layer which stands for Step 2 showed different behavior 

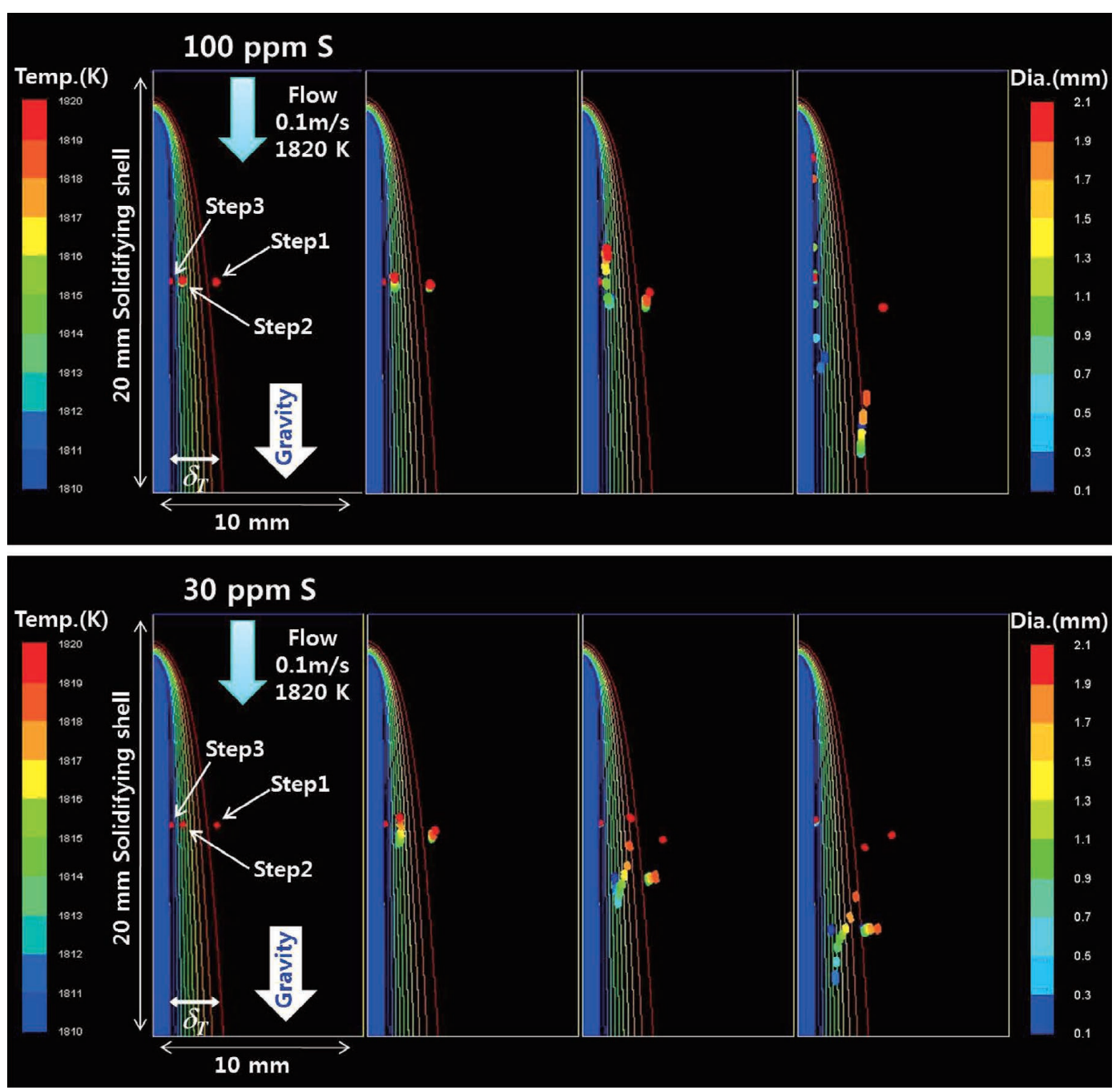

Fig. 13. FLUENT simulation results of case $30 \mathrm{~S}$ and $100 \mathrm{~S}$.

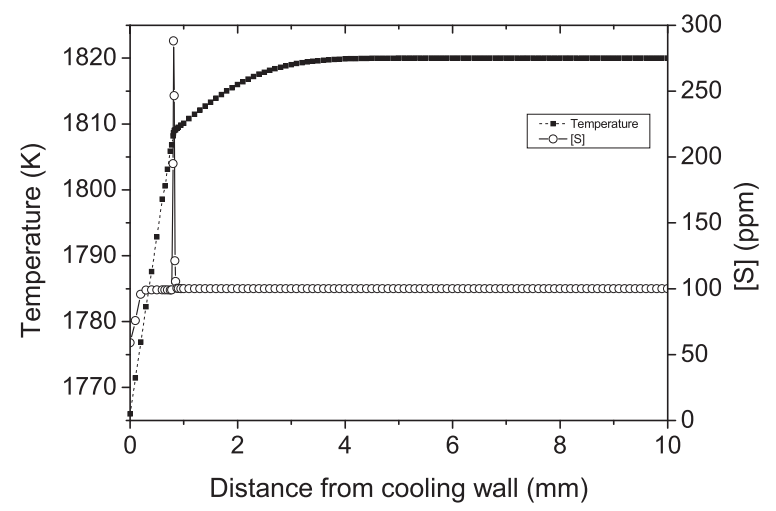

Fig. 14. Profiles of the temperature and sulfur concentration.

according to the sulfur concentration. The bubbles are pushed away against the solidifying shell in the case of $30 \mathrm{ppm}$ of bulk sulfur concentration, and pulled into it in the case of $100 \mathrm{ppm}$. Hence, the thermal marangoni force is generated ahead of the solutal one and it determines the direction of bubble movement in the vicinity of the solidifying interface. The third group outside of boundary layers which stands for Step 1 moved only up and down depending on their size since small bubble was swept down by the drag force of the fluid flow and large bubble float up by the buoy- ancy force.

As a result, the motion of bubble is changed by the sulfur concentration in the thermal boundary layer at Step 2, but the most of bubbles are entrapped regardless of the sulfur concentration in the solutal boundary layer at Step 3. Hence, the result of simulation in Fig. 11 validates that the motion of the bubble at the $\mathrm{S} / \mathrm{L}$ interface is critically affected by the thermal marangoni force in the thermal boundary layer before it reaches in the solutal boundary layer.

\section{Conclusions}

The mechanism which governs the behavior of bubbles in the vicinity of the solidification front where both the concentration and temperature gradients exist was investigated and the effect of the solutal and thermal marangoni forces to the entrapment of bubble were compared and discussed.

(1) In front of the solid/liquid interface during steel casting, there exist two different boundary layers which are most influential on the behavior of bubbles. These are the thermal and concentration boundary layers. As the thermal boundary layer is much thicker than the concentration boundary layer, bubbles in the molten steel may enter into the boundaries, but into the thermal boundary layer first.

(2) The direction of the thermal marangoni force acting 
on the bubble in the thermal boundary layer is changed by the critical value of the sulfur concentration. The critical value was found to be in the range of 47-60 ppm for temperature of $1809-1840 \mathrm{~K}\left((\partial \sigma / \partial T)_{C}<0\right.$ when $C_{S}<47-60 \mathrm{ppm}$, and $(\partial \sigma / \partial T)_{C}>0$ when $\left.C_{S}>47-60 \mathrm{ppm}\right)$. When a bubble enters into the thermal boundary layer, hence, it will be pushed away from the solidification front when the sulfur concentration is lower than the critical value so that it will have no chance to be attached at the solid/liquid interface. When the sulfur concentration is higher than the critical value, on the other hand, the bubble will be pulled toward the solidification front in the thermal boundary layer, and eventually enter the concentration boundary layer where a strong pulling force will exert to the bubble so that the bubble will then be attached onto the solid/liquid interface.

(3) 2D CFD simulation was conducted in order to validate the above analysis. The forces acting on the surface of a bubble computed by the simulation clearly revealed the pulling or pushing force depending on temperature for a given sulfur concentration. All of the above analyses were in good agreement with data obtained from a plant trial.

\section{REFERENCES}

1) H. Yasunaka, R. Yamanaka, T. Inoue and T. Saito: Tetsu-to-Hagané, 80 (1995), 529

2) H. Shibata, H. Yin, S. Yoshinaga, T. Emi and M. Suzuki: ISIJ Int., 38 (1998), 149
3) S. Kimura, Y. Nabeshima, K. Nakajima and S. Mizoguchi: Metall. Mater. Trans. B, 31B (2000), 1013.

4) D. M. Stefanescu, F. R. Juretzko, B. K. Dhindaw, A. Catalina, S. Sen and P. A. Curreri: Metall. Mater. Trans. A, 29A (1998), 1697.

5) F. R. Juretzko, B. K. Dhindaw, D. M. Stefanescu, S. Sen and P. A. Curreri: Metall. Mater. Trans. A, 29A (1998), 1691.

6) H. Ohta and H. Suito: ISIJ Int., 46 (2006), 472.

7) H. Yasuda, I. Ohnaka, T. Yano and H. Tanaka: ISIJ Int., 36 (1996), S167.

8) B. G. Thomas, A. Dennisov and H. Bai: Proc. of ISS 80th Steelmaking Conf., ISS, Warrendale, PA, (1997), 375.

9) Q. Yuan, B. G. Thomas and S. P. Vanka: Metall. Mater. Trans. B, 35B (2004), 703

10) Q. Yuan and B. G. Thomas: Proc. of 3rd Int. Cong. on Sci. \& Tech. of Steelmaking, AIST, Warrendale, PA, (2005).

11) K. Mukai and W. Lin: Tetsu-to-Hagané, 80 (1994), 527.

12) Z. Wang, K. Mukai and I. J. Lee: ISIJ Int., 39 (1999), 553.

13) K. Mukai and W. Lin: Tetsu-to-Hagané, 80 (1994), 533

14) K. Mukai and M. Zeze: Steel Res. Int., 74 (2003), 131.

15) K. Mukai, L. Zhong and M. Zeze: ISIJ Int., 46 (2006), 1810.

16) T. Miyake, M. Morishita, H. Nakata and M. Kokita: ISIJ Int., 46 (2006), 1817.

17) G. R. Belton: Metall. Mater. Trans. B, 7B (1976), 35.

18) P. Sahoo, T. Debroy and M. J. McNallan: Metall. Mater. Trans. B, 19B (1988), 483.

19) B. J. Keene: Int. Mater. Rev., 33 (1988), 1.

20) T. Nishi, H. Shibata, H. Ohta and Y. Waseda: Metall. Mater. Trans. A, 34A (2003), 2801.

21) W. A. Tiller, K. A. Jackson, J. W. Rutter and B. Chalmers: Acta Metall., 1 (1953), 428

22) N. Kasai, H. Mizukami and A. Mutou: Tetsu-to-Hagané, 89 (2003), 28

23) T. Toh, H. Hasegawa and H. Harada: ISIJ Int., 41 (2001), 1245.

24) H. Schlichting: Boundary Layer Theory, 7th ed., McGraw-Hill, New York, (1979), 306.

25) M. C. Flemings: Solidification Processing, McGraw-Hill, New York, (1974), 41. 